\title{
Effective pedagogy as a local experience
}

\author{
Margaret Walshaw
}

Published online: 12 June 2014

(C) Springer Science+Business Media Dordrecht 2014

There is always a lot of discussion about effective pedagogical practices in mathematics. While we might vary in our views about the detail, those of us based in western countries are generally agreed on approaches that emphasize student centeredness and inclusiveness. For example, we understand that effective teachers work hard at developing trusting classroom communities. We believe that, in turn, the relationships developed within that community become a resource for developing students' mathematical competencies and identities. Effective teachers have high yet realistic expectations about what their students can achieve. They ensure that the lesson content has a strong mathematical focus and contains opportunities for students to think, reason, communicate, reflect upon and critique mathematics.

However, how relevant for other settings are our western understandings of effective mathematics pedagogy? We sometimes assume that pedagogical practices that have shown to be effective in one setting will be effective for every school system, every community and every student cohort. That assumption appears to underwrite the exportation of tests and textbooks that find their way into developing countries. In essence, the practice represents a one-way transfer of expertise and materials through which the game of western 'catch-up' is played. Some researchers have been drawing attention to this point. They argue that pedagogical decisions in non-western settings should be grounded in local knowledge, language, culture, family systems and values and beliefs. Engagement with all these realities would unravel modes of thinking and acting within the local social order that have important implications for teaching practices.

The three papers in this issue each report on research undertaken within different international settings. They explore prospective teacher learning and self-perceptions from locations based in Ethiopia, Samoa and South Africa. The researchers do not dismiss the developments on pedagogy identified in the literature, but they filter those developments

M. Walshaw ( $\square)$

Massey University, Palmerston North, New Zealand

e-mail: m.a.walshaw@massey.ac.nz 
through an engagement with their respective contexts, cultures and histories. They do that at a level that will make a difference both to students and to the community. In brief, they give expression to a two-way transfer of ideas and practices, through which sustainable educational futures might become achievable.

From within their setting in Ethiopia, Hailu Nigus Weldeana and Solomon Tewolde Abraham wanted to explore the effects of a history-based intervention on prospective middle-grade (5-8) teachers' beliefs about mathematics. In "The Effect of an Historical Perspective on Prospective Teachers' Beliefs in Learning Mathematics," the researchers describe an intervention that provided participants a means to explore applications of mathematical problems in real life and was intended to enhance their understandings of the historical development of mathematics ideas. The researchers used pretest and posttest Likert-type questionnaires as well as written reflections and observations of participants' oral presentations to assess any change in their beliefs about mathematics as a result of the semester-long intervention.

The participants worked on problem-solving activities in small groups but wrote their reflective accounts individually. Problems included applications of the Fibonacci sequence, of Euclid's Method and of Pythagoras's theorem and required participants, as one participant explained, "to discover and figure everything out on my own way, instead of waiting for someone to do or explain for me." In a cultural setting in which mathematics is often assumed to be a male domain, the intervention also initiated a shift in thinking toward a recognition of the contribution females have made to mathematics over history. The findings also provided evidence of a number of prospective teachers' shift from a focus on memorizing mathematics toward a focus on understanding mathematics. On the basis of their findings, the researchers make a case for the use of context-rich problems from the history of mathematics that are able to be customized for problem-solving activities relating to fundamental mathematical topics. In opposition to customary practice within the country in which the research took place, the authors emphasize that teacher interventions should be minimal and facilitative. They also caution that shifting beliefs is difficult work and that prospective teachers sometimes fail to appreciate that interventions are designed to enhance mathematical understanding.

In a different international setting and through the development of meaningful and contextualized mathematics learning experiences within a semester-long methods course, Karoline Afamasaga-Fuata'i and Lumaava Sooaemalelagi, based in Samoa, focused on enhancing prospective teachers' mathematical understandings and attitudes. In "Student Teachers' Mathematics Attitudes, Authentic Investigations and Use of Metacognitive Tools," the researchers explain how the methods course aligned with a new official curriculum that had moved away from traditional approaches toward a characterization of teaching that embraced mathematical communication and the development of critical thinking, reasoning and analytical skills. The participants in the study were students repeating their first year primary mathematics program and a number of other students, with poor records of mathematical achievement. Course instructors provided support and scaffolding as the students were introduced to and experienced the curriculum reform. During workshops, the prospective teachers constructed concept maps to demonstrate their understanding and also produced Venn diagrams to highlight their solution processes and the underlying principles.

Data from the questionnaire responses collected at the beginning and end of the methods course were analyzed for 53 and 45 prospective teachers, respectively, using the Partial Credit Rasch Model to assess prospective teachers' competence. Interview data gathered at the end of the course were analyzed qualitatively to establish factors that contribute to 
attitudinal change. Findings revealed that solving a mathematical problem by independent means positively influenced attitudes toward mathematics. On the other hand, for some prospective teachers, feelings of discomfort, impatience, and anxiety, and a view of mathematics as memorization became entrenched by the end of the methods course. Like the researchers in the first study in this issue, the researchers in the Samoan context provide evidence of the difficult task involved in shifting understandings of and attitudes toward mathematics.

In yet another international context, this time in Pretoria, South Africa, researchers Sonja van Putten, Gerrit Stols and Sarah Howie sought to gain insight into the professional identity of prospective teachers. Professional teacher identity, they maintain, is a complex and multifaceted phenomenon. In "Do Prospective Mathematics Teachers Teach who They say They are?" professional teacher identity is theorized as constituted by different aspects of identity (named as sub-identities) that come to the fore in specific contexts and within specific relationships. These sub-identities are explained as represented by teachers' views of themselves as specialists in mathematics content knowledge and skills, as specialists in relation to teaching and learning, and as carers. With this framework, the researchers explored whether prospective teachers' understanding of their professional teacher identity coincided with the teacher identity that was enacted within the classroom.

The six participants in the study were drawn from prospective teachers enrolled in a fourth year course preparing them to become mathematics specialists and teach higher secondary school grade levels. As part of the course, the prospective teachers spent one half of their program on teaching practice (internship). Individual interviews were conducted prior to the commencement of the practicum and had been designed to add depth to the exercise prospective teachers had undertaken to provide a ranking of themselves, and an explanation of that ranking, in relation to subject specialist, teaching-and-learning specialist and carer. In addition, two lessons taught by each prospective teacher were videotaped. The ranking exercise, the interviews and the classroom observations were all subsequently coded, and when analyzed, revealed that the prospective teachers' views of themselves as specialists in content, teaching and learning and as carers were not always the same as gleaned from their teaching. That is to say, espoused theory and enacted theory were not always aligned. The researchers suggest that lack of experience may contribute to a false sense of professional identity. They also propose that a professional mathematics teacher identity may well be context driven. Since in this study talking about and enacting a teaching identity occurred in different contexts, the researchers suggest that a professional mathematics teaching identity might be more adequately explained by prospective teachers within the context of their teaching.

As the researchers in this issue have shown, research does not always give us the answers we anticipate. The prospective teachers in the study undertaken in South Africa all had a sound mathematical background and were learning how to teach senior students. Yet their perceptions of their own teaching, in most cases, missed the mark. Similarly, the studies carried out in Ethiopia and in Samoa were developed with the best interests of prospective students in mind. Initiatives and courses designed to enhance teachers' knowledge and understanding of mathematics, do not, as the studies reported here demonstrate, always produce the outcomes desired. This is not a new phenomenon. It is simply to make us aware of the need for further research and further explanation. 